\title{
Correction to: Incidental intracranial meningiomas: a systematic review and meta-analysis of prognostic factors and outcomes
}

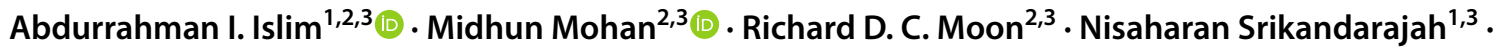 \\ Samantha J. Mills ${ }^{4}$ Andrew R. Brodbelt ${ }^{3}$ Michael D. Jenkinson ${ }^{1,3}$ (1)
}

Published online: 31 July 2019

(c) The Author(s) 2019

\section{Correction to: \\ Journal of Neuro-Oncology (2019) 142:211-221 \\ https://doi.org/10.1007/s11060-019-03104-3}

Issues with data analysis have recently been highlighted by a reader of our article. These have been addressed with changes to Tables 2 and 4, as shown below, and Online Resources 5-7. T2 and peritumoral signal are no longer prognostic factors on simple pooled (Online Resource 5) and IPD (Table 4) analyses respectively. In Table 5, the number of patients which informed the outcomes symptom development and intervention were 575 and 947 respectively; 69 developed symptoms (pooled proportion \%8.4 [95\% CI 2.8-16.7], $\mathrm{I}^{2}=88.9 \%$ ). These included motor and cognitive deficits $(n=1)$. We apologise to the readership of the Journal of Neuro-Oncology for these errors and thank the reader for helping us identify them.

Abdurrahman I. Islim, Midhun Mohan and Richard D. C. Moon contributed equally to the manuscript.

The original article can be found online at https://doi.org/10.1007/ s11060-019-03104-3.

Electronic supplementary material The online version of this article (https://doi.org/10.1007/s11060-019-03237-5) contains supplementary material, which is available to authorized users.

Abdurrahman I. Islim

a.islim@liv.ac.uk

1 Institute of Translational Medicine, University of Liverpool, Liverpool, UK

2 Faculty of Health and Life Sciences, University of Liverpool, Liverpool, UK

3 Department of Neurosurgery, The Walton Centre NHS Foundation Trust, Liverpool, UK

4 Department of Neuroradiology, The Walton Centre NHS Foundation Trust, Liverpool, UK
Open Access This article is distributed under the terms of the Creative Commons Attribution 4.0 International License (http://creativeco mmons.org/licenses/by/4.0/), which permits unrestricted use, distribution, and reproduction in any medium, provided you give appropriate credit to the original author(s) and the source, provide a link to the Creative Commons license, and indicate if changes were made.

Publisher's Note Springer Nature remains neutral with regard to jurisdictional claims in published maps and institutional affiliations. 
Table 2 Baseline clinical and radiological characteristics

\begin{tabular}{|c|c|c|c|c|c|c|c|c|}
\hline $\begin{array}{l}\text { No. of studies } \\
\text { informing char- } \\
\text { acteristic }\end{array}$ & $\begin{array}{l}\text { No. of valid } \\
\text { cases informing } \\
\text { characteristic }(\%)\end{array}$ & Characteristics & & Total & Surgery & SRS & $\begin{array}{l}\text { Active monitor- } \\
\text { ing }\end{array}$ & $\mathrm{P}$ \\
\hline 18 & 2050 & N. of patients $(\%)$ & & 2050 & $560(27.3)$ & $450(22.0)$ & $1040(50.7)$ & \\
\hline 13 & $803(39.2)$ & $\begin{array}{l}\text { Mean age, years } \\
\text { (SD) }\end{array}$ & & $63.1(6.9)$ & $61.5(4.7)$ & $54.9(\mathrm{NR})^{\mathrm{b}}$ & $64(6.9)$ & $<0.001$ \\
\hline \multirow[t]{2}{*}{17} & $1948(95.0)$ & Sex, N (\%) & Female & 1549 & $369(23.8)$ & $363(23.4)$ & 817 (52.7) & 0.539 \\
\hline & & & Male & 399 & $89(22.3)$ & $87(21.8)$ & $223(55.9)$ & \\
\hline \multirow[t]{11}{*}{16} & $1495(72.9)$ & Location, $\mathrm{N}(\%)^{\mathrm{a}}$ & Non-skull base & 1024 & $277(27.1)$ & 235 (22.9) & $512(50.0)$ & $<0.001$ \\
\hline & & & Convexity & 431 & 126 & 86 & 219 & \\
\hline & & & Parafalcine & 245 & 61 & 71 & 113 & \\
\hline & & & Parasagittal & 151 & 42 & 36 & 73 & \\
\hline & & & Tentorial & 62 & 12 & 28 & 22 & \\
\hline & & & Intraventricular & 26 & 4 & 12 & 10 & \\
\hline & & & Skull base & 471 & $106(22.5)$ & $154(32.7)$ & $211(44.8)$ & \\
\hline & & & Anterior midline & 112 & 22 & 43 & 47 & \\
\hline & & & Sphenoid wing & 98 & 25 & 11 & 62 & \\
\hline & & & $\begin{array}{l}\text { Posterior fossa } \\
\text { - lateral and } \\
\text { posterior }\end{array}$ & 52 & 23 & 12 & 17 & \\
\hline & & & $\begin{array}{l}\text { Posterior fossa - } \\
\text { midline }\end{array}$ & 138 & 17 & 87 & 34 & \\
\hline 15 & $888(43.3)$ & $\begin{array}{l}\text { Mean diameter, } \\
\mathrm{cm}(\mathrm{SD})\end{array}$ & & $2.14(0.61)$ & $2.11(0.42)$ & $1.73(\mathrm{NR})^{\mathrm{b}}$ & $2.19(0.66)$ & $<0.001$ \\
\hline \multirow[t]{2}{*}{10} & $615(30.0)$ & $\begin{array}{l}\text { Calcification, } \mathrm{N} \\
(\%)\end{array}$ & No & 394 & $64(16.2)$ & NR & $330(83.8)$ & 0.177 \\
\hline & & & Yes & 221 & $27(12.2)$ & NR & $194(87.8)$ & \\
\hline \multirow[t]{2}{*}{5} & $298(14.5)$ & $\begin{array}{l}\text { Tumor signal } \\
\text { intensity, } \mathrm{N}(\%)\end{array}$ & Hyperintense & 120 & $40(33.3)$ & NR & $80(66.6)$ & 0.237 \\
\hline & & & Iso/hypointense & 178 & $48(27.0)$ & NR & $130(73.0)$ & \\
\hline \multirow[t]{2}{*}{12} & $1107(54.0)$ & $\begin{array}{l}\text { Peritumoral } \\
\text { edema, N (\%) }\end{array}$ & Yes & 144 & 47 (32.6) & $24(16.7)$ & $73(50.7)$ & \\
\hline & & & No & 963 & $145(15.1)$ & 365 (37.9) & $453(47.0)$ & \\
\hline
\end{tabular}

$N R$ not reported, $S R S$ stereotactic radiosurgery

${ }^{a}$ One study which dichotomized location into supratentorial and infratentorial was excluded [15]

${ }^{\mathrm{b}}$ Available in one study which did not report SD [25] 
Table 4 Growth dynamics and symptom development during active monitoring stratified by baseline characteristics

\begin{tabular}{|c|c|c|c|c|c|c|c|c|}
\hline Factor & & $\begin{array}{l}\text { Mean } / \text { median } \\
\text { AGR }\left(\mathrm{cm}^{3} /\right. \\
\text { year })\end{array}$ & $\mathrm{P}$ & $\begin{array}{l}\text { Mean/median } \\
\text { RGR (\%/year) }\end{array}$ & $P$ & $\begin{array}{l}\text { Symptom develop- } \\
\text { ment, yes/total (\%) }\end{array}$ & OR $(95 \% \mathrm{CI})$ & MLR Pa \\
\hline \multirow[t]{2}{*}{ Location } & Non-skull base & $7.97 / 0.68$ & 0.747 & $70.2 / 17.0$ & 0.405 & $12 / 60(20.0)$ & & 0.116 \\
\hline & Skull base & $5.82 / 2.93$ & & $28.1 / 17.2$ & & $5 / 25(20.0)$ & & \\
\hline \multirow[t]{2}{*}{ Diameter } & $\geq 3.0 \mathrm{~cm}$ & $19.7 / 5.91$ & 0.004 & $114 / 24.7$ & 0.091 & $15 / 27(55.5)$ & $12.16(5.56-18.78)$ & $<0.001$ \\
\hline & $<3.0 \mathrm{~cm}$ & $1.56 / 0.46$ & & $31.4 / 12.7$ & & $2 / 58(3.45)$ & & \\
\hline \multirow[t]{2}{*}{ Calcification } & No & $19.0 / 19.0$ & NA & $141 / 141$ & NA & $1 / 1(100)$ & & \\
\hline & Yes & NR & & NR & & NR & & \\
\hline \multirow[t]{2}{*}{ Tumor signal intensity } & Hyperintense & NR & NA & NR & NA & NR & & \\
\hline & Iso/hypointense & NR & & NR & & NR & & \\
\hline \multirow[t]{2}{*}{ Peritumoral edema } & Yes & NR & NA & NR & NA & NR & & \\
\hline & No & $2.32 / 0.62$ & & $19.7 / 13.2$ & & $1 / 29(3.45)$ & & \\
\hline
\end{tabular}

$A G R$ absolute growth rate, $R G R$ relative growth rate, $M L R$ multi-level regression, $N A$ not applicable, $N R$ not reported

${ }^{\text {a}}$ Performed to 50 iterations 